\title{
ASYMPTOTIC VALUES OF AN ENTIRE FUNCTION AND ITS DERIVATIVE
}

\author{
BO KJELLBERG and BENGT SANDSTRÖM
}

1. Asymptotic values. Let us consider entire functions $f(z)$ of a complex variable $z$. We set $F(r)=\max |f(z)|$ on $|z|=r$ and define the order $\varrho$ and type $\tau$ by

$$
\varrho=\limsup _{r \rightarrow \infty} \frac{\log \log F^{\prime}(r)}{\log r}, \quad \tau=\limsup _{r \rightarrow \infty} \frac{\log F(r)}{r^{0}} .
$$

A function of finite positive order $\varrho$ is said to be of minimum, mean or maximum type if $\tau=0,0<\tau<\infty$ or $\tau=\infty$ respectively.

Let $\Gamma$ be a curve in the $z$-plane which stretches out towards infinity. Suppose $f(z)$ has a finite limit $a$ as $z \rightarrow \infty$ along $\Gamma$. Then $a$ is said to be an asymptotic value of the function $f(z)$. On this topic much has been written since the conjecture of Denjoy in 1907, that the number $n$ of asymptotic values is bounded by $n \leq 2 \varrho$. This fact was proved more than 20 years later by Ahlfors, Beurling, Carleman and others.

2. What happens to tho derivative? A number of authors have later investigated the following problem. Question: Let $a$ be a finite number and suppose that $f(z) \rightarrow a$ as $z \rightarrow \infty$ along a curve $\Gamma$. Is it then necessarily true that $f^{\prime}(z) \rightarrow 0$ as $z \rightarrow \infty$ along $\Gamma$ ?

Jaenisch [4] has made an extensive study of this question, earlier taken up by Plancherel, Pólya, Delange, Gaier and others. Functions up to order $1 / 2$, minimum type, have no finite asymptotic values because their minimum modulus is unbounded according to the classical theorem by Wiman.

However, for functions of order $1 / 2$, mean type, the answer to the question is yes. The same holds for functions up to order 1, minimum type, if $\Gamma$ is a straight line or has a curvature $O(1 /|z|)$.

For functions with growth at least of order 1, mean type, the answer to the question is no. 
3. The scope of this investigation. From what has been said, it remains to investigate functions of orders from $1 / 2$ to 1 if arbitrarily curved paths $\Gamma$ are allowed. For each $\varrho$ in this interval we intend to construct an entire function of order $\varrho$ and a path $I$ which is asymptotic for $f(z)$ but not for $f^{\prime}(z)$.

Thus for functions of order $\varrho>1 / 2$ the answer to the question posed is no.

It still remains to study a limiting case, functions of order $1 / 2$, maximum type. We indicate an analogous construction in this case also.

4. An earlier construction. One of the present authors, Sandström, did succeed already several years ago in constructing suitable functions in the case $1 / 2<\varrho<1$.

From what has been said it follows that it is useless to work with rectilinear or weakly curved paths. Therefore, the paths chosen were of the type here illustrated in Figure 1.

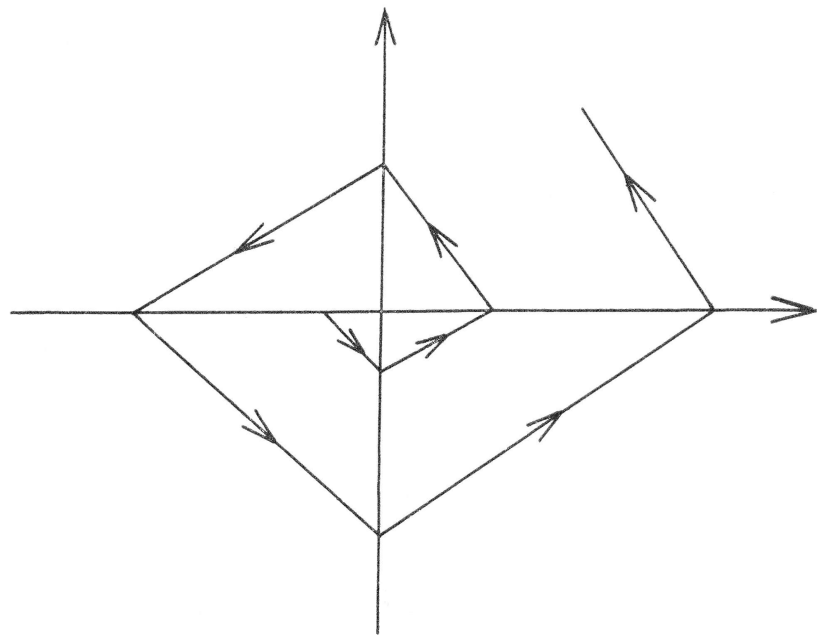

Figure 1.

It turned out that it was possible to construct entire functions $f(z)$ such that $f(z) \rightarrow 0$ along $\Gamma$ but not $f^{\prime}(z)$. The latter had large values at the corners. One thing, however, which was felt to be unsatisfactory, was that the exact value of the order $\varrho$ could not be stated. Only by a continuity argument was it possible to infer that a suitable function could be obtained for each order $\varrho, 1 / 2<\varrho<1$.

Our intention now is to obtain a direct construction for given $\varrho$.

5. The method of construction. As a first step we intend to construct a subharmonic function $u(z)$ resembling $\log |f(z)|$, where $f(z)$ 
is the desired entire function. A subharmonic function has associated with it a certain mass distribution in the plane which is equal to $\Delta u$ taken in the sense of distribution theory. In a second step we move the mass a little in order to form unit masses.

In such a manner $\log |f(z)|$ and then $f(z)$ is obtained. The method has been used earlier, see, for instance Kjellberg [5, Ch. 4], Hayman [2].

6. Construction of the subharmonic function. Let $\varrho$ be given, $1 / 2<\varrho<1$. Set $z=r e^{i \vartheta}$ and consider the two rays $\vartheta=\pi \mp \pi / 2 \varrho$, starting from the points with real part 1 , which are connected by a line segment.

On the ray $\vartheta=\pi-\pi / 2 \varrho$ we take a number of points $P_{1}, P_{2}, \ldots, P_{n} \ldots$ at distances $R_{1}, R_{2}, \ldots, R_{n}, \ldots$ from the origin. It might be good to have $R_{n} / R_{n-1}>4$.

Let us, for instance, take

$$
R_{n}=e^{2 n}, \quad n=1,2, \ldots .
$$

From each $P_{n}$ we draw upwards a line segment $P_{n} Q_{n}$ of length $l_{n}$, perpendicular to the ray $\vartheta=\pi-\pi / 2 \varrho$.

Let us take

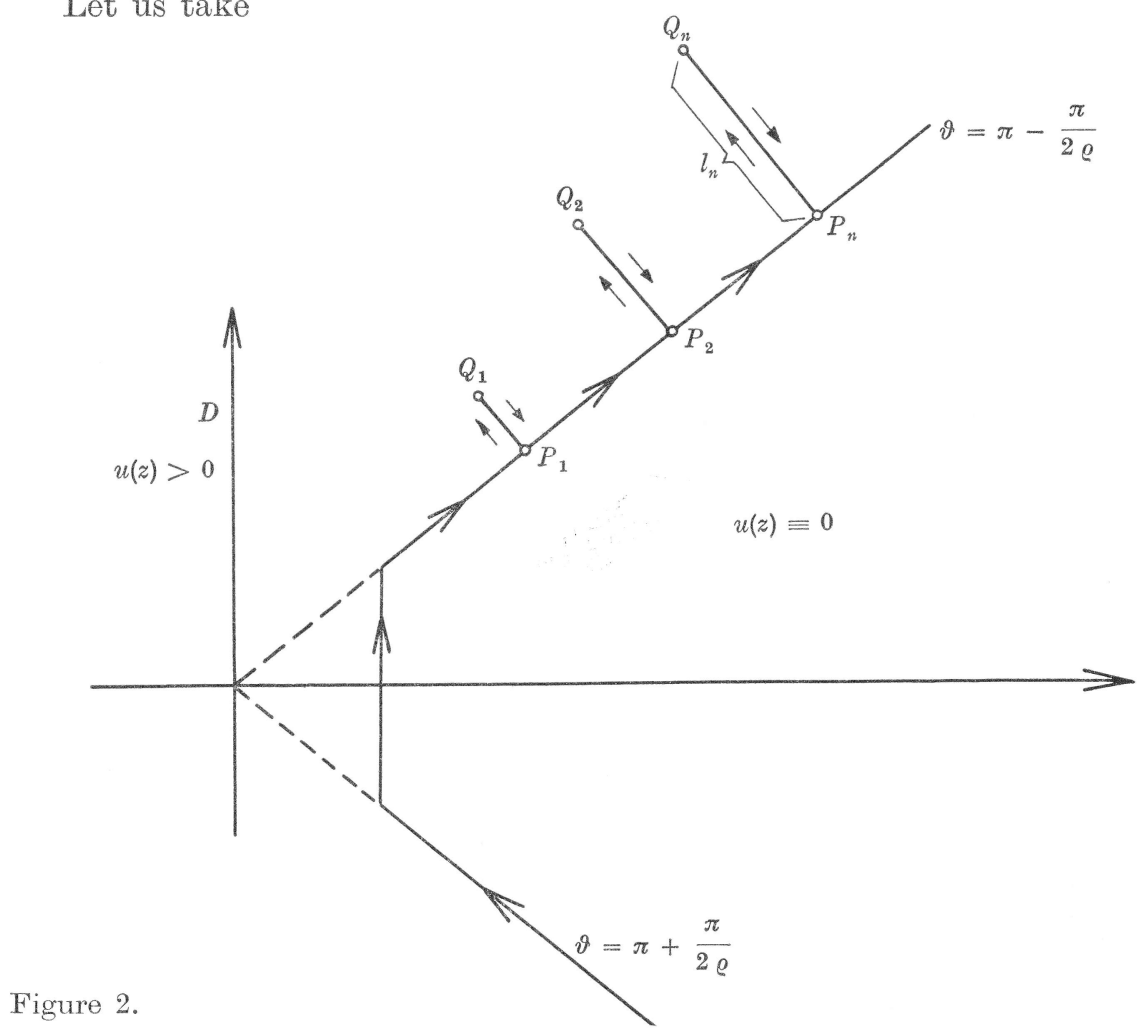




$$
l_{n}=\frac{R_{n}}{\left(\log R_{n}\right)^{2}}=\frac{e^{2 n}}{4 n^{2}}
$$

so that the sum of $l_{n} / R_{n}$ is convergent, a fact which will be used later.

The three segments first constructed and all the segments $P_{n} Q_{n} P_{n}$ constitute the boundary $\Gamma$ of a simply connected region $D$ containing the part of the real axis to the left of $z=1$.

We can now define the subharmonic function $u(z)$. Let $u(z)$ be harmonic and positive in $D$, let $u(0)=+1$ and $u(z)=0$ on the boundary $\Gamma$. Then $u(z)$ exists uniquely in $D$, a fact well-known for a half-plane upon which $D$ can be mapped. For a very simple proof, see Tideman [7]. In the rest of the plane we set $u(z)=0$. The function $u(z)$ so constructed is subharmonic and non-negative in the whole plane.

If $\Gamma$ simply were the rays $\vartheta=\pi \mp \pi / 2 \varrho$ and $u(-1)=+1$, then $u(z)$ should be $r^{\varrho} \cos \varrho(\vartheta-\pi)$ in the left part of the plane. Now $u(z)$ is somewhat different, a few properties are listed in Section 9.

7. Construction of the entire function. The function $u(z)$, as defined above, has the representation, see, e.g. Heins [3],

$$
u(z)=1+\int_{\Gamma} \log \left|1-\frac{z}{t}\right| d \mu(t) .
$$

Here $\mu(t)$ is a function monotonically increasing as $t$ traverses $T$ in the direction indicated in Figure 2.

Let us set $\mu(1)=0$. We remember that $d \mu(t)$ in the integral stands for $(1 / 2 \pi)(\partial u / \partial n)|d t|$, where $\partial u / \partial n$ is the derivative in the direction of the normal pointing into $D$.

We now move the mass a little by setting

$$
h(z)=1+\int_{\Gamma} \log \left|1-\frac{z}{t}\right| d[\mu(t)]
$$

where $[\mu(t)]$ signifies the integral part of $\mu(t)$. We could just as well write

$$
h(z)=1+\sum_{1}^{\infty} \log \left|1-\frac{z}{t_{p}}\right|
$$

where $t_{y}, v=1,2, \ldots$, signify the discontinuity points of $[\mu(t)]$, ordered with respect to their distances from the origin.

We shall show in Sections 10 and 11 that

$$
-25 \log |z|<h(z)-u(z)<25 \log |z|
$$


for $|z|$ sufficiently large. The left inequality, however, holds only if the distance from $z$ to $T$ is at least $1 /|z|$.

Let us now define

$$
f(z)=\sum_{28}^{\infty}\left(1-\frac{z}{t_{\nu}}\right)
$$

It then holds with good margin for large $|z|$ that

$$
-53 \log |z|<\log |f(z)|-u(z)<-\log |z|
$$

with the aforementioned restriction for the left inequality. We show in 9a that $f(z)$ is of order $\varrho$. Because $u(z)=0$ on $\Gamma$, it is seen from (7.6) that $f(z) \rightarrow 0$ as $z \rightarrow \infty$ along $\Gamma$.

8. Study of $f^{\prime}(z)$. Let us fix a point $Q_{n}$ (see Figure 2) and study $|f|$ and $\left|f^{\prime}\right|$ near this point. We define a nearby point $Q_{n}^{\prime}$ as follows. If already $\left|f^{\prime}\left(Q_{n}\right)\right| \geq 1$ we set $Q_{n}^{\prime}=Q_{n}$. If $\left|f^{\prime}\left(Q_{n}\right)\right|<1$ the same inequality $\left|f^{\prime}(z)\right|<1$ holds on some interval on the prolongation of $P_{n} Q_{n}$. For each $z$ on this interval we have

$$
\left|f(z)-f\left(Q_{n}\right)\right|=\left|\int_{Q_{n}}^{z} f^{\prime}(\zeta) d \zeta\right| \leq\left|z-Q_{n}\right|=\delta .
$$

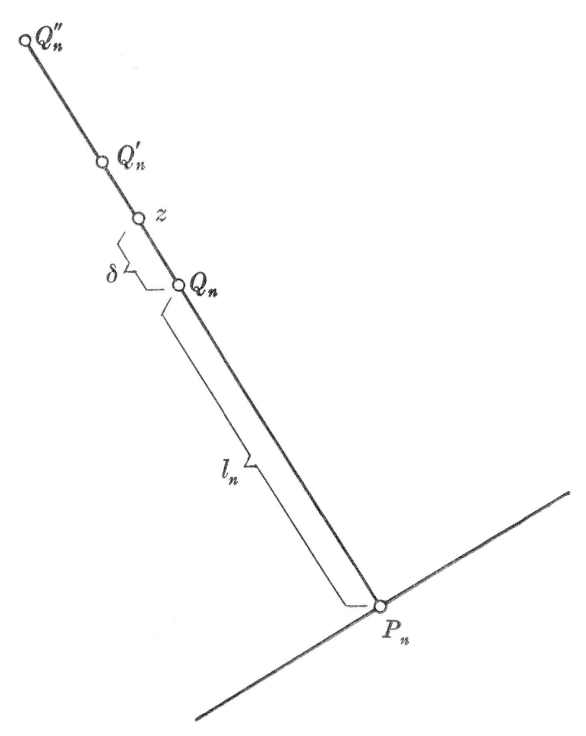

Figure 3 , 
Because $\left|f\left(Q_{n}\right)\right|<1 / R_{n}$ by (7.6) we obtain, since $u\left(Q_{n}\right)=0$,

$$
|f(z)| \leq\left|f\left(Q_{n}\right)\right|+\delta<\frac{1}{R_{n}}+\delta .
$$

The left inequality in (7.6) gives for $\delta>1 / R_{n}$ that

$$
\log |f(z)|>u(z)-53 \log |z|>\frac{\sqrt{\delta}}{150} \frac{R_{n}^{\varrho-1 / 2}}{\log R_{n}}-53 \log R_{n} .
$$

Here we use an estimate of $u(z)$ which is obtained in $9 \mathrm{~d}$. It is obvious that the distance $\left|z-Q_{n}\right|$, i.e. $\delta$, must be very short for this to hold. If we compare (8.1) and (8.2) and denote by $Q_{n}^{\prime}$ the first point on the prolongation of $P_{n} Q_{n}$ for which $\left|f^{\prime}\left(Q_{n}^{\prime}\right)\right|=1$, we see that surely $\left|Q_{n}^{\prime}-Q_{n}\right|<R_{n}^{-(\varrho-1 / 2)}$, for instance. It follows from (8.1) that $|f(z)| \rightarrow 0$ uniformly on $Q_{n} Q_{n}^{\prime}$ as $n \rightarrow \infty$.

Let us now define a certain path $\Gamma^{\prime}$. It starts from $z=1$ and follows $\Gamma$ to the point $P_{1}$. Then it makes a detour $P_{1} Q_{1}^{\prime} P_{1}$, thereafter it follows $\Gamma$ to $P_{2}$, makes a detour $P_{2} Q_{2}^{\prime} P_{2}$, goes from $P_{2}$ to $P_{3}$, makes a detour $P_{3} Q_{3}^{\prime} P_{3}$ and so on.

From the construction it follows that $f(z) \rightarrow 0$ as $z \rightarrow \infty$ along $\Gamma^{\prime}$. But this is not true for $f^{\prime}(z)$. At the cusps $Q_{n}^{\prime}$ of $\Gamma^{\prime}$ we have $\left|f^{\prime}\left(Q_{n}^{\prime}\right)\right| \geq 1$, but from the comments to $9 \mathrm{c}$ we infer that $u(z),|f(z)|$ and thus $\left|f^{\prime}(z)\right|$ are close to zero in a wide strip around the segments $P_{n} P_{n+1}$ which are parts of $\Gamma^{\prime}$.

To sum up, we have constructed an entire function $f(z)$ of order $\varrho$, $1 / 2<\varrho<1$, and a path $\Gamma^{\prime}$, asymptotic for $f(z)$ but not for $f^{\prime}(z)$.

9. Properties of $u(z)$. Let us study $u(z)$ in Section 6 , harmonic and positive in the region $D$, zero on the boundary $\Gamma$ and in the rest of the plane. We need some facts about $u(z)$.

9a. $u(z)$ is of order $\varrho(u(z)$ corresponds to $\log |f(z)|$ in the definition of order in Section 1).

9b. $u(z)$ takes its maximum $M(r)$ on $|z|=r$ near $z=-r$.

9c. $u(z) \rightarrow 0$ as $z \rightarrow \infty$ in the strip consisting of those $z$ whose distance from $T$ is at most $1 /|z|$.

9d. $u(z)$ grows rapidly near $Q_{n}$, if $z$ is located as indicated in Figure 3 one has

$$
u(z) \geq \frac{\sqrt{\delta}}{150} \frac{R_{n}^{\varrho-1 / 2}}{\log R_{n}}
$$

for sufficiently large $n$. As before, $R_{n}=\left|P_{n}\right|$. 
Comments to 9a. There are formulae giving a lower estimate of the growth of the maximum modulus $M(r)$ in $D$. Theorem 7.6 in Fuchs [1] gives for $n>1$ that

$$
M(r) \geq \frac{M(1)}{4} \exp \left\{\pi \int_{1}^{r} \frac{d t}{t \vartheta(t)}\right\}>\frac{1}{4} r^{\varrho} .
$$

In our case $M(1)>1$ and the angular measure $\vartheta(t)$ of that part of $|z|=t$ which is contained in $D$, is $\leq \pi / \varrho$.

On the other hand there must for each $\sigma>\varrho$ exist $r_{\sigma}$ such that

$$
M(r) \leq r^{\sigma} \quad \text { for } r \geq r_{\sigma} .
$$

For suppose that there exists a sequence $r_{1}, r_{2}, \ldots \rightarrow \infty$ such that $M\left(r_{n}\right)>r_{n}^{\sigma}$. Choose $\tau$ such that $\varrho<\tau<\sigma$. Because of (6.2) there exists $r_{\tau}$ such that the region $D_{\tau}$, defined by $\pi-\pi / 2 \tau<\arg z<$ $\pi+\pi / 2 \tau,|z|>r_{\tau}$, is contained in $D$. Let $C_{\tau}^{n}$ denote the arc in $D_{\tau}$ of $|z|=r_{n}$. Because $r_{n}^{\sigma}<M\left(r_{n}\right)=u\left(r_{n} e^{i \vartheta_{n}}\right)$, where $\vartheta_{n} \approx \pi$ by $9 \mathrm{~b}$, there exists by Harnack's theorem a constant $C_{\tau}>0$ such that $u(z)>$ $C_{\tau} r_{n}^{\sigma}$ for $z \in C_{\tau}^{n}$. If $z \in D_{\tau}$ and $|z|<r_{n}$ then

$$
u(z)>C_{\tau} r_{n}^{\sigma-\tau}\left(r^{\tau}-r_{\tau}^{2 \tau} r^{-\tau}\right) \cos \tau(\vartheta-\tau) .
$$

The right-hand expression is a harmonic minorant of $u(z)$ having boundary values $\leq C_{\tau} r_{n}^{\sigma}$ on $C_{\tau}^{n}$, zero elsewhere. In each point of $D_{\tau}$ the minorants $\rightarrow+\infty$ as $n \rightarrow \infty$. Thus (9.3) must hold.

Comments to $9 \mathrm{~b}$. By transformation $w=\log z$ that part of $D$ where $|z|>1$ is mapped into a half-strip of breadth $\pi / \varrho$. The growth of $u$ towards the middle of the strip can be shown simply by reflection in circles.

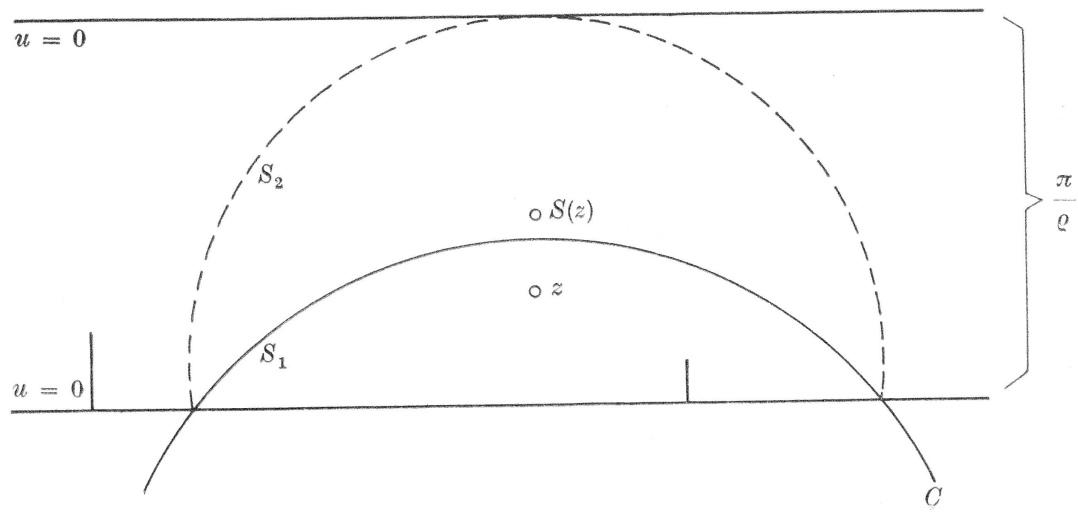

Figure 4, 
The procedure is indicated in Figure 4. The points $z$ and $S(z)$ are symmetric with respect to the circle $C$. For each $C$ chosen so that the image $S_{2}$ of $S_{1}$ stays in the strip where $u(z)>0$ it holds that $u\{S(z)\}-u(z)>0$ if $z \in S_{1}$.

If $M(r)=u\left(r e^{i \vartheta}\right)$ it follows that $\vartheta \rightarrow \pi$ as $r \rightarrow \infty$.

Comments to $9 \mathrm{c}$. Take a point $\zeta$ on a segment $P_{n} P_{n+1}$ such that $R=|\zeta|$ exceeds $r_{\sigma}$ defined in (9.3). Construct a semicircle above the diameter with end points $\zeta / 2$ and $3 \xi / 2$. Form the harmonic majorant of $u(z)$ equal to zero on the diameter, $(3 R / 2)^{\sigma}$ on the arc. Then (see, e.g., Nevanlinna [6, Ch. 3, §2]) for $|z-\zeta| \leq d<R / 2$,

$$
u(z) \leq \frac{4}{\pi} \arctan \frac{d}{R / 2} \cdot\left(\frac{3}{2} R\right)^{\sigma} \leq 4 d R^{-1+\sigma} .
$$

Thus $u(z) \rightarrow 0$ as $z \rightarrow \infty$ in a wide strip around the ray $\vartheta=\pi-$ $\pi / 2 \varrho$. Also, $\partial u / \partial n \rightarrow 0$ as $z \rightarrow \infty$ along this ray. The same holds for the ray $\vartheta=\pi+\pi / 2 \varrho$. In order to estimate $u(z)$ in the neighbourhood of the segments $P_{n} Q_{n}$ we consider a circle centered at $Q_{n}$ and with radius $2 l_{n}$. We obtain a majorant of $u(z)$ on the circumference from (9.5) by choosing $d=3 l_{n}, \quad R=R_{n}$, which gives $u(z) \leq 12 l_{n} R_{n}^{-1+\sigma}$. On the radius through $P_{n}$ we have $u(z)=0$ by definition. By $w=\left(z-Q_{n}\right)^{1 / 2}$ the cut circle is transformed into a half-circle. If the distance from $z$ to $P_{n} Q_{n}$ is $\leq \delta$ we obtain, remembering (6.2), that

$$
u(z) \leq \frac{4}{\pi} \arctan \frac{\sqrt{\delta}}{\sqrt{2 l_{n}}} \cdot 12 l_{n} \cdot R_{n}^{-1+\sigma} \leq 12 \sqrt{\delta} \frac{R_{n}^{\sigma-1 / 2}}{\log R_{n}} .
$$

We have here omitted the little discussion that shows that the most rapid growth of $u(z)$ takes place near $Q_{n}$. By taking $\delta=1 / R_{n}$ the validity of $9 \mathrm{c}$ also near $P_{n} Q_{n}$ is proved.

Comments to $9 \mathrm{~d}$. Let us go to Figure 3 and denote by $Q_{n}^{\prime \prime}$ the point beyond $Q_{n}^{\prime}$ whose distance to $Q_{n}$ is $l_{n} / 4$. By (9.2) $M\left(R_{n}\right) \geq R_{n}^{o} / 4$.

Make for a moment the logarithmic transformation as in the comments to $9 \mathrm{~b}$. By Harnack's theorem for a circle we roughly obtain

$$
u\left(Q_{n}^{\prime \prime}\right)>\frac{\varrho}{5 \pi} \frac{l_{n}}{R_{n}} M\left(R_{n}\right)>\frac{1}{40 \pi} l_{n} R_{n}^{-1+\varrho}
$$

for sufficiently large $n$. Go back to Figure 3 , set $w=\left(z-Q_{n}\right)^{1 / 2}$ and apply Harnack's theorem taking a circle with radius $\sqrt{l_{n}} / 2$, centered at the image point of $Q_{n}^{\prime \prime}$. Then, for $z$ as in Figure 3,

$$
u(z) \geq \frac{\sqrt{\delta}}{\sqrt{l}_{n}} u\left(Q_{n}^{\prime \prime}\right) \geq \frac{\sqrt{\delta}}{150} \frac{R_{n}^{\varrho-1 / 2}}{\log R_{n}} .
$$

We see that $\partial u / \partial n \rightarrow \infty$ at $Q_{n}$. 
10. The displacement of the masses. Let $z$ be a point not on $\Gamma$ and $|z|>2$. From (7.1) and (7.2) we infer that

$$
h(z)-u(z)=\int_{\Gamma} \log \left|1-\frac{z}{t}\right| d\{[\mu(t)]-\mu(t)\} .
$$

A partial integration gives that

$$
h(z)-u(z)=-\int_{\Gamma}\{[\mu(t)]-\mu(t)\} d \log \left|1-\frac{z}{t}\right|
$$

which gives

$$
|h(z)-u(z)| \leq \int_{\Gamma}|d \log | 1-\frac{z}{t}||
$$

In the next section we prove that the integral in (10.2) is less than $25 \log |z|$ if $|z|$ is large enough and if the distance from $z$ to $\Gamma$ is at least $1 /|z|$. To prove (7.4) it remains to prove that

$$
h(z)-u(z)<25 \log |z|
$$

holds also if $z$ is close to $\Gamma$.

Let us restrict ourselves to the half-plane $\operatorname{Re} z>2$ and denote by $E$ the set of $z$ in this half-plane whose distance to $\Gamma$ is less than $1 /|z|$. We have just seen that the subharmonic function $h(z)-25 \log |z|<u(z)$ on the boundary of $E$ if $|z|$ is large enough. Because $u(z) \rightarrow 0$ on this boundary as $|z| \rightarrow \infty$ (proved in 9c) there exists a constant $K$ such that $h(z)-25 \log |z|<K$ on the boundary. If we set

$$
v(z)=\left\{\begin{array}{l}
\max \{h(z)-25 \log |z|, K\} \quad \text { for } z \in E, \\
K \text { elsewhere, }
\end{array}\right.
$$

we obtain a function which is subharmonic in the half-plane considered and equal to $K$ on its boundary. The order of $h(z)$ and then $v(z)$ is $<1$ because if follows from the form of (7.3) that the maximum modulus of $h(z)$ on $|z|=r$ is taken in the left half-plane where $h(z)$ is closely connected to $u(z)$ by (10.2) which has order $\varrho<1$.

By the theorem of Phragmén-Lindelöf it follows that $v(z) \leq K$ in the half-plane. In particular for $z \in E$ we obtain $h(z)-25 \log |z|<K$ and since $u(z) \geq 0$ also $h(z)-u(z)<25 \log |z|+K$. As we see in next section that the number 25 could be chosen somewhat smaller, the validity of (10.3) is obvious and also (7.4).

11. The variation of the logarithm. We want to estimate the integral in (10.2) but begin with an arbitrary straight line instead of $\Gamma$. Let us 
fix $z,|z|>2$, and denote by $L$ the set of $t$ on the line where $|t|>1$ and $|t-z|>1 /|z|$. By the Möbius transformation $w=1-z / t$ the circles $|t|=1$ and $|t-z|=1 /|z|$ will, for large $|z|$, be mapped approximately on $|w|=|z|$ and $|w|=1 /|z|^{2}$. When $t$ runs through $L$ the corresponding point $w$ will run through those parts of a line or a circle which are situated in the ring between the two circles mentioned. That means that the variation of $\log |w|$ can be $\approx 6 \log |z|$ at most, i.e.

$$
\int_{\Gamma}|d \log | 1-\frac{z}{t}|| \approx 6 \log |z|
$$

at most for large $|z|$.

We also need a corresponding estimate for a short line segment such as $P_{n} Q_{n}$, i.e.

$$
\int_{P_{n}}^{Q_{n}}|d \log | 1-\frac{z}{t}|| \leq \int_{P_{n}}^{Q_{n}}\left|d \log \left(1-\frac{z}{t}\right)\right|=\int_{P_{n}}^{Q_{n}} \frac{|z||d t|}{|t||t-z|} .
$$

On $P_{n} Q_{n}$, whose length is $l_{n}$, we have $|t| \approx R_{n}$. If we know that $|t-z|>|z| / 2$ then the integral in (11.2) can be $\approx 2 l_{n} / R_{n}$ at most.

We are now in a position to estimate the variation of $\log |1-z| t \mid$ on $\Gamma$ supposing that the distance from $z$ to $T$ is $1 /|z|$ at least. If there is a $P_{n} Q_{n}$ in the ring $|z| / 2<t<3|z| / 2$ the variation on $P_{n} Q_{n} P_{n}$ can be $\approx 12 \log |z|$ at most.

On each one of the rays $\vartheta=\pi \mp \pi / 2 \varrho$ the variation is $\approx 6 \log |z|$ at most. The sum of the variations on all remaining line segments is bounded by a fixed constant. Thus

$$
\int_{\Gamma}|d \log | 1-\frac{z}{t}|| \leq 25 \log |z|
$$

for sufficiently large $|z|$; the coefficient could be taken somewhat less than 25 .

12. Some final comments. We formulated a question in Section 2 . The answer is yes for functions of order $1 / 2$, mean type, the answer is no for functions of order $\varrho>1 / 2$. Thus there is a limiting case to investigate, namely functions of order $1 / 2$, maximum type. To obtain them in our manner we have to replace the rays $\vartheta=\pi \mp \pi / 2 \varrho$ by curves closer to the positive real axis. The growth is estimated as was done in 9a. Let us write $M(r)=A(r) r^{1 / 2}$ where $\lim \sup A(r)=\infty$ as $r \rightarrow \infty$. In (8.2) the factor $R_{n}^{o-1 / 2}$ will be replaced by $A\left(R_{n}\right)$. In order to succeed with the 
same sort of proof we need that $A\left(R_{n}\right)$ surpasses some multiple of $\left(\log R_{n}\right)^{2}$. We leave open the question of what is true in this connection for functions having a weaker growth within the class of functions of order $1 / 2$, maximum type.

Let us at last mention that the $E_{\alpha}$-functions of Mittag-Leffler could be used for our constructions. These functions, together with all the derivatives, tend to zero in an angular region. Suppose we have constructed as described here above one suitable function $f(z)$ of order $\varrho$ and a path $\Gamma$. If we take $\sigma$ such that $\varrho<\sigma<1$, then $F^{\prime}(z)=f(z)+E_{1 / \sigma}(-z)$ is an example of a function of order $\sigma>\varrho$ with $F(z) \rightarrow 0$ along $\Gamma$ but not $F^{\prime}(z)$.

\section{References}

[1] Fuchs, W. H. J.: Topics in the theory of functions of one complex variable. D. Van Nostrand Company, Inc., Princeton, N. J.-Toronto, Ont.London, 1967.

[2] Hayman, W. K.: On integral functions with distinct asymptotic values. - Proc. Cambridge Philos. Soc. 66, 1969, 301-315.

[3] HEINS, M.: Entire functions with bounded minimum modulus; subharmonic function analogues. - Ann. of Math. (2) 49, 1948, 200-213.

[4] JAENISCH, S.: Abschätzungen subharmonischer und ganzer Funktionen in der Umgebung einer Kurve. - Nitteilungen aus dem Mathem. Seminar Giessen, Giessen 1965.

[5] KJEIIBERG, B.: On certain integral and harmonic functions. - Thesis, Uppsala, 1948.

[6] Nevanimna, R.: Eindeutige analytische Funktionen. - Die Grundlehren der mathematischen Wissenschaften 46, 2:e Aufl., Springer-Verlag, BerlinGöttingen-Heidelberg, 1953.

[7] TIDEMAN, M.: Elementary proof of a uniqueness theprem for positive harsonic functions. - Nordisk Mat. Tidskr. 2, 1954, 95-98.

Kungl. Tekniska Högskolan

Institutionen för Matematik

S-100 44. Stockholm 70

Sweden

Received 26 October 1976
Universitetsfilialen

Fakultetsgatan 1

70130 Örebro

Sweden 